Acknowledgements Supported by a Pilot \& Feasibility Grant from the George M. O'Brien Center for Kidney Research at Yale (NIH P30 DK079310, J.C.), grants from the NIH R37 AR40072 (J.C.), R01 AR074545 (J.C.), and R21 AI142145 (J. C), NIH U19 AI082724 (M.R.C), and AbbVie (J.C.). P.M.C. was the recipient of government scholarship for graduate study from the Ministry of Education, Taiwan, and Gershon Fellowship from the Department of Immunobiology, Yale University.

\section{DEVELOPING A STANDARDIZED STEROID DOSING REGIMEN IN PEDIATRIC PROLIFERATIVE LUPUS NEPHRITIS}

${ }^{1}$ Hermine I Brunner* ${ }^{*}{ }^{2} \mathrm{~K}$ Rouster-Stevens, ${ }^{3}$ Marisa S Klein-Gitelman, ${ }^{4}$ Karen Onel, ${ }^{5}$ Beatrice Goilav, ${ }^{6}$ Natasha Ruth, ${ }^{1}$ Tingting Qiu, ${ }^{1}$ Najla Aljaberi, ${ }^{7}$ Jianghong Deng, ${ }^{8}$ Benjamin L Laskin, ${ }^{9}$ Anna Carmela P Sagcal-Gironella, ${ }^{10}$ Stacy P Ardoin, ${ }^{11}$ Deborah M Levy, ${ }^{9}$ Scott E Wenderfer, 'Bin Huang. 'Cincinnati Children's Hospital Medical Center, Cincinnati, Ohio, USA; 'Emory University, Children's Healthcare of Atlanta, Atlanta, Georgia, USA; ${ }^{3}$ Northwestern University Feinberg School of Medicine, Ann and Robert H Lurie Children's Hospital of Chicago, Chicago, IL, USA; ${ }^{4}$ Division of Pediatric Rheumatology, Hospital for Special Surgery, New York, NY, USA; ${ }^{5}$ The Children's Hospital at Montefiore, Albert Einstein College of Medicine, Bronx, NY, USA; ${ }^{6}$ Medical University of South Carolina, Charleston, South Carolina, USA; ${ }^{7}$ Beijing Children's Hospital, Capital Medical University, National Center for Children's Health, Beijing, China; ${ }^{8}$ Children's Hospital of Philadelphia, Perelman School of Medicine, University of Pennsylvania, Philadelphia, PA, USA; ${ }^{9}$ Department of Pediatrics, Baylor College of Medicine, Houston, Texas, USA; ${ }^{10}$ Nationwide Children's Hospital, Columbus, Ohio, USA; ${ }^{11}$ The Hospital for Sick Children and The University of Toronto, Toronto, Ontario, Canada

\subsection{6/lupus-2021-lupus21century.21}

Background Corticosteroids (CS) remain the mainstay of therapy for childhood-onset systemic lupus erythematosus (cSLE) although there are no widely accepted dosing strategies of oral (PO CS) or intravenous CS (IV CS). We aimed to (1) develop a standardized CS dosing regimen (SSR) and (2) achieve consensus for this SSR among pediatric rheumatology and nephrology providers treating cSLE complicated by lupus nephritis (LN).

Methods Consensus formation techniques were used. A Delphi questionnaire pertaining to CS use in cSLE was completed to inform formats of the Patient Profiles (PP, Step 1). Using data from 147 children with proliferative $\mathrm{LN}$ at 8 major cSLE

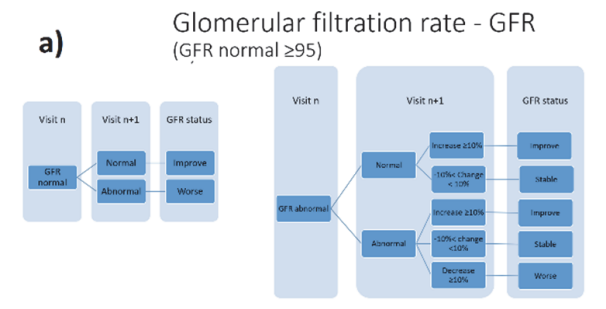

c) Glomerular hematuria - RBC/HPF

(categories: normal 0-5, 6-10, 11-25, 26-50, >50)

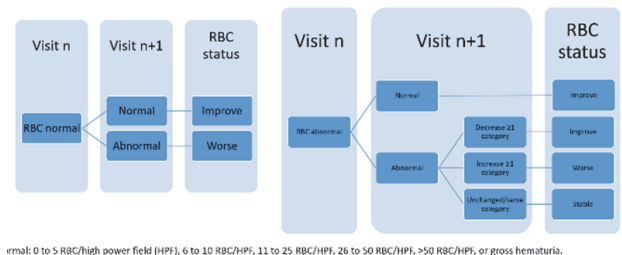

treatment sites in North America PP were generated providing information about the course of $\mathrm{LN}$ and extra-renal cSLE (ER) at 2 subsequent visits (Step 2). PP were sent to 142 physicians (PP-raters) experienced in cSLE to adjudicate the course of ER and LN and propose the PO/IV CS dosages (Step 3). Using data from PP for which consensus was achieved, the SSR was developed (Step 4) and refined based on responses from another questionnaire and a focus group of experienced physicians (Step 5). The SSR was tested using a second type of PP that described ER and LN courses for 6 months from the time of biopsy (Step 6). Consensus was defined as agreement of the majority of PP-raters (Step 3, Step 6).

Results For Step 1 and Step 3, 103 physicians answered Delphi questions and filled 353 PP (response rate: 73\%). Step 6 activities were completed by 18 physicians (13.4 years of average experience) who were asked to review 33 PP each. This resulted in 564 completed PP ratings, of which 437 $(77.5 \%)$ and $460(81.6 \%)$ ratings yielded consensus on POSSR-and IV SSR dosing, respectively. PO CS and/or IV CS dosages as per the SSR (SSR-dose) depend on patient weight, the course of ER activity measured by the ER-SLEDAI score (figure 1d), and the course of LN described by changes/status of $3 L N$ response variables ( $L N-R V s$, figure 1a-c). The SSR mimics dosing customs agreed upon by the PP-raters. Table 1 summarizes the SSR with focus on $2 \mathrm{ER} /$ LN settings (1:stable ER/various LN courses; 2:stable LN/ various ER courses), with several permutation of the course of ER (much worse, mild-moderately worse, active stable/ improved, inactive) and LN (flare, mild-moderately worse, active stable/improved/partial renal remission (PRR), complete renal remission (CRR)). Use of dosages of PO CS $\geq$ $40 \mathrm{mg}$ are governed by the course of $\mathrm{LN}$ except in major ER flares with potential organ damage. The SSR adjusts PO CS dosages in at least monthly intervals. IV CS are used for worsening of ER or LN courses that fail to respond to increased oral CS of $\geq 40 \mathrm{mg}$ up to 4 weeks. Small decreases of PO CS occur even with stable ER or stable LN activity. Achieving CRR leads to more pronounced reduction of PO CS (table 1). Beyond 6 months post kidney biopsy (maintenance therapy), the PO/IV CS dosage is informed by LN status (PRR, CRR), the course of LN and ER activity (table 1).

b) Urine protein creatinine ratio - UPCR (UPCR normal $\leq 0.2$ )

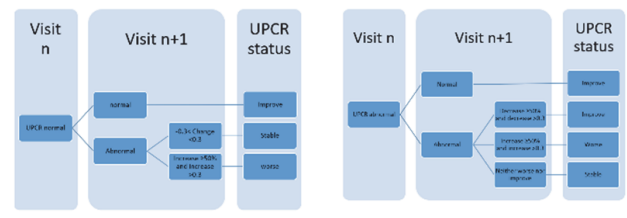

d) ER Course

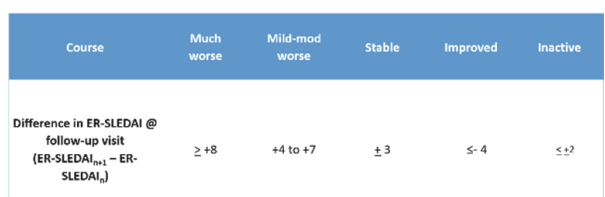


Abstract 503 Table 1 Steroid use provided by the standardized steroid regimen (SSR)

INITIAL 4 WEEKS OF INDUCTION THERAPY

\begin{tabular}{|c|c|c|}
\hline \multicolumn{2}{|r|}{ PO CS } & IV CS \\
\hline Patients $\geq 50 \mathrm{~kg}$ & Prednisone* $60 \mathrm{mg} /$ day divided in up to 4 doses & Up to 3 doses (30 mg/kg; max 1 gram of methylprednisolone) \\
\hline Patients < $50 \mathrm{~kg}$ & Prednisone $1.5 \mathrm{mg} / \mathrm{kg} / \mathrm{day}$ & \\
\hline $\begin{array}{l}\text { Median - lowest PO CS } \\
\text { dose at week } 4^{* *}\end{array}$ & $40 \mathrm{mg} /$ day - $30 \mathrm{mg} /$ day & \\
\hline \multicolumn{3}{|c|}{ WEEK 5 - 26 OF INDUCTION THERAPY (based on LN and ER trends since last visit) } \\
\hline LN course & Much worse & Increase PO CS to $50-60 \mathrm{mg} /$ day; re-assess in $1-3$ weeks; if response to increased PO CS is (a) \\
\hline \multirow[t]{6}{*}{ (assumption ER is stable) } & & Satisfactory $\rightarrow$ No IV CS; (b) non-satisfactory $\rightarrow$ IV pulses $+P O C S$ \\
\hline & & Possible change of immunosuppressive drug \\
\hline & Mild - moderately worse & Increase PO CS by about $30 \%$ (if dose < $40 \mathrm{mg}$; max $60 \mathrm{mg}$ ) \\
\hline & Active stable & Stable PO CS dose (if dose < 40 mg; else: slow decrease) \\
\hline & Improved active or $P R R^{1}$ & Slow decrease of PO CS dose \\
\hline & $C R R^{2}$ & More pronounced decrease of PO CS dose \\
\hline \multirow[t]{5}{*}{$\begin{array}{l}\text { ER course } \\
\text { (assumption } L N \text { is stable) }\end{array}$} & Much worse & $\begin{array}{l}\text { Increase PO CS dose; Re-assess in 1-3 weeks; if response to increased PO CS dose is (a) Satisfactory } \rightarrow \\
\text { No IV CS; (b) non-satisfactory } \rightarrow I V \text { pulses + PO CS dose; }\end{array}$ \\
\hline & & Possible change of immunosuppressive drug \\
\hline & Mild- moderately worse & Increase PO CS by $20 \%$ for doses < $40 \mathrm{mg}$; otherwise stable PO CS dose \\
\hline & Active stable or improved active & Stable PO CS dose \\
\hline & Inactive & Decrease PO CS dose \\
\hline $\begin{array}{l}\text { Median - Lowest PO CS } \\
\text { dose possible at week } 26\end{array}$ & $12.5-10 \mathrm{mg} /$ day & \\
\hline \multicolumn{3}{|c|}{ BEYOND 26 WEEKS POST KIDNEY BIOPSY - MAINTENANCE THERAPY } \\
\hline LN course & Flare $^{3}$ after PRR/CRR & Prednisone $\geq 40 \mathrm{mg}$, irrespective of ER course \\
\hline \multirow[t]{4}{*}{ (assumption ER is stable) } & & $\begin{array}{l}\text { Re-assess in 1-3 weeks; if response to increased PO CS is (a) Satisfactory } \rightarrow \text { No IV CS; (b) non- } \\
\text { satisfactory } \rightarrow I V \text { pulses + PO CS }\end{array}$ \\
\hline & Worse after PRR/CRR & Increase the PO CS dose FIRST \\
\hline & PRR stable & Slow decrease of the SSR-dose \\
\hline & Inactive/CRR or PRR improved & More pronounced decrease of the SSR dose \\
\hline \multirow[t]{4}{*}{$\begin{array}{l}\text { ER course (assumption PRR } \\
\text { parameters are stable) }\end{array}$} & Much worse & $\begin{array}{l}\text { Increase PO CS dose by } 30-50 \% \text { (max } 60 \mathrm{mg} \text { ); Re-assess in } 1-3 \text { weeks; if response to increased PO CS } \\
\text { dose is (a) Satisfactory } \rightarrow \text { No IV CS ; (b) non-satisfactory } \rightarrow I V \text { pulses + PO CS; }\end{array}$ \\
\hline & & Possible change of immunosuppressive drug \\
\hline & Mild- moderately worse & Increase PO CS dose by $25 \%$ for doses < 40 mg; otherwise stable PO CS dose \\
\hline & Stable/Improved/Inactive & Decrease of the PO CS dose \\
\hline
\end{tabular}

${ }^{* *}$ For patients $\geq 50 \mathrm{~kg} ;{ }^{*}$ or corticosteroid equivalent dose.

${ }^{1}$ Partial renal remission (PRR): $>50 \%$ improvement of $\geq 2$ LN-RVs PLUS remaining LN-RV is NOT worse.

${ }^{2}$ Complete renal remission (CRR): All LN-RVs are NORMAL.

${ }^{3} \mathrm{LN}$ flare defined by at least 1 of the LN-RV changes being persistently present on $\geq 2$ subsequent time points $\geq 1$ week apart. LN-RV changes are defined as (a) newly abnormal GFR, (b) abnormal GFR that decreased by $>10 \%$, (c) persistent increase of UPCR to $\geq 0.5$, after CRR, (d) persistent doubling of UPCR with values $\geq 1.0$, after PRR, or (e) newly active or worsening glomerular hematuria.

Conclusions SSR for the treatment of cSLE complicated by LN has been developed which simulates PO/IV CS use among treating physicians. The proposed SSR may be useful for clinical care and to regulate background CS use during clinical trials of new medication for cSLE.

Acknowledgments Presented on behalf of the LaUNCH Project Investigator and supported by PORTICO (P30AR076316).

\section{SPECIFIC IN SITU INFLAMMATORY ARCHITECTURES PREDICT PROGRESSION TO RENAL FAILURE IN HUMAN LUPUS NEPHRITIS}

Madeleine Durkee, Rebecca Abraham, Junting Ai, Anthony Chang, Kichul Ko, Maryellen Giger, Marcus R Clark*. University of Chicago, IL, USA

\subsection{6/lupus-2021-lupus21century.22}

Background In human lupus nephritis (LN), tubulointerstitial inflammation (TII) on biopsy predicts refractory disease and progression to end stage renal disease (ESRD). However, while approximately half of patients with moderate or severe TII develop ESRD, half do not. Therefore, we hypothesized that TII is heterogeneous with distinct inflammatory states each associated with different renal outcomes.

Methods We interrogated renal biopsies from LN longitudinal (55 patients) and cross-sectional cohorts using both conventional and highly-multiplex (24 analytes) confocal microscopy. To accurately segment cells across whole biopsies, and to understand their spatial relationships, we trained and implemented a suite of computer vision tools, including multiple parallel Mask-R convolutional neural networks. This evolution of our previous analytic pipeline, Cell Distance Mapping (CDM)(Nat Immunol, 2019, 20:503), we refer to as CDM version 4.

Results Across biopsies, B cell densities were strongly associated with protection from ESRD ( $\mathrm{p}=2 \times 10^{-8}$, Mann-Whitney). In contrast, CD4- $\mathrm{T}$ cell population densities, which included CD8, gd and double negative (CD4-CD8-, DN) T cells, predicted progression to ESRD $\left(\mathrm{p}=5.7 \times 10^{-16}\right)$. Breath first search and other analyses revealed inflammation was 